\title{
Maps preserving numerical radius distance on $\mathrm{C}^{*}$-algebras
}

\author{
by \\ Zhaofang Bai (Xi'an and Linfen), Jinchuan Hou (Linfen and Taiyuan) \\ and ZongBen XU (Xi'an)
}

\begin{abstract}
We characterize surjective nonlinear maps $\Phi$ between unital $\mathrm{C}^{*}$-algebras $\mathcal{A}$ and $\mathcal{B}$ that satisfy $w(\Phi(A)-\Phi(B))=w(A-B)$ for all $A, B \in \mathcal{A}$ under a mild condition that $\Phi(I)-\Phi(0)$ belongs to the center of $\mathcal{B}$, where $w(A)$ is the numerical radius of $A$ and $I$ is the unit of $\mathcal{A}$.
\end{abstract}

1. Introduction. In the middle forties of the twentieth century, L. K. Hua initiated the study of geometry of matrices. The fundamental problem of geometry of matrices is to characterize the group of motions by as few geometric invariants as possible [8]. Hua discovered that, for some fields $\mathbb{F}$, especially the real field $\mathbb{R}$ and complex field $\mathbb{C}$, the "adjacency" invariant ( $T$ and $S$ are adjacent if $\operatorname{rank}(T-S)=1$ ) alone is sufficient to characterize the motions (up to automorphisms of the underlying field) on spaces of matrices, symmetric matrices, skew-symmetric matrices and hermitian matrices, respectively. Motivated by the geometry of matrices, a similar fundamental question may be raised for the infinite-dimensional case.

Problem. Find as few as possible properties that may be possessed by operator spaces $\mathcal{A}$ and $\mathcal{B}$ or by elements in them and that are enough to determine the structure of the map $\Phi: \mathcal{A} \rightarrow \mathcal{B}$ if $\Phi$ has these properties as invariants, i.e., if $\Phi$ preserves these properties.

Thus the problem is indeed to develop an analog of "geometry of matrices" for operators. For $\mathcal{B}(H)$, where $H$ is an infinite-dimensional Hilbert space and $\mathcal{B}(H)$ is the von Neumann algebra of all bounded linear operators on $H$, we proved in [1] that the invariant "numerical radius distance" alone is sufficient to characterize the group generated by the following five simple kinds of maps (motions):

2000 Mathematics Subject Classification: Primary 47B49, 47A12; Secondary 47H20.

Key words and phrases: numerical radius distance, $\mathrm{C}^{*}$-algebra.

This work is supported partially by NNSFC and PNSFS. 
(1) $A \mapsto U A U^{*}$, where $U \in \mathcal{B}(H)$ is a unitary operator;

(2) $A \mapsto U A U^{*}$, where $U: H \rightarrow H$ is a conjugate unitary operator;

(3) $A \mapsto A^{*}$;

(4) $A \mapsto \mu A$, where $\mu \in \mathbb{C}$ and $|\mu|=1$;

(5) $A \mapsto A+S$, where $S \in \mathcal{B}(H)$.

It was shown in [3] that "numerical radius distance" is also an invariant which is enough to characterize surjective nonlinear maps between atomic nest algebras. The purpose of this paper is to characterize maps preserving numerical radius distance on $\mathrm{C}^{*}$-algebras.

Let $\mathcal{A}$ and $\mathcal{B}$ be unital $\mathrm{C}^{*}$-algebras. Denote by $I$ and $I^{\prime}$ the units of $\mathcal{A}$ and $\mathcal{B}$, respectively. Recall that a state $\tau$ on $\mathcal{A}$ is a positive linear functional on $\mathcal{A}$ with $\tau(I)=1$. For $A \in \mathcal{A}$, the numerical range and numerical radius of $A$ are defined, respectively, by

$$
\begin{aligned}
W(A) & =\{\tau(A) \mid \tau \text { runs over all states on } \mathcal{A}\}, \\
w(A) & =\sup \{|\lambda| \mid \lambda \in W(A)\} .
\end{aligned}
$$

It is well known that numerical radius, $w(\cdot)$, is a norm (but not a $\mathrm{C}^{*}$-norm) and is equivalent to the original norm on $\mathcal{A}$. A map $\Phi: \mathcal{A} \rightarrow \mathcal{B}$ (no linearity assumed) is numerical distance preserving if $w(\Phi(A)-\Phi(B))=w(A-B)$ for all $A, B \in \mathcal{A}$. We also recall the definition of $\mathrm{C}^{*}$-isomorphism and conjugate $\mathrm{C}^{*}$-isomorphism. A bijective map $\Phi: \mathcal{A} \rightarrow \mathcal{B}$ is a $\mathrm{C}^{*}$-isomorphism if $\Phi$ is linear and satisfies

$$
\begin{aligned}
& \Phi\left(A^{*}\right)=\Phi(A)^{*}, \\
& \Phi\left(A^{2}\right)=\Phi(A)^{2}
\end{aligned}
$$

for every $A \in \mathcal{A}$ (i.e., $\Phi$ is a *-Jordan isomorphism); $\Phi$ is a conjugate $\mathrm{C}^{*}$ isomorphism if $\Phi$ is conjugate linear (i.e., $\Phi$ is additive and $\Phi(\lambda A)=\bar{\lambda} \Phi(A)$ for every $A \in \mathcal{A}$ and every complex number $\lambda$ ) and satisfies (1.1) and (1.2). From the classical Mazur-Ulam theorem [6], it follows that every numerical radius distance preserving surjective map sending 0 to 0 is real-linear. Thus we essentially deal with the real-linear numerical radius preserving maps on $\mathrm{C}^{*}$-algebras. Our main result (Theorem 1) shows that every unital surjective real-linear numerical radius preserving map is a direct sum of a $\mathrm{C}^{*}$-isomorphism and a conjugate $\mathrm{C}^{*}$-isomorphism. From our main result, we also get some corollaries which characterize the surjective numerical radius distance preserving maps between some special $\mathrm{C}^{*}$-algebras such as von Neumann algebras and von Neumann algebra factors.

Throughout this paper, the center of a $\mathrm{C}^{*}$-algebra $\mathcal{A}$ is the set $\mathcal{Z}(\mathcal{A})=$ $\{A \in \mathcal{A} \mid A B=B A$ for all $B \in \mathcal{A}\}$. An element $P \in \mathcal{A}$ is called a projection if $P^{2}=P$ and $P^{*}=P$, and a central projection if $P$ is a projection and $P \in \mathcal{Z}(\mathcal{A})$. An element $A \in \mathcal{A}$ is called a partial isometry if $A^{*} A$ and $A A^{*}$ are projections; in particular, $A$ is a unitary element if $A^{*} A=A A^{*}=I$. A map 
$\Phi: \mathcal{A} \rightarrow \mathcal{B}$ is said to be numerical radius preserving if $w(\Phi(A))=w(A)$ for every $A \in \mathcal{A}$.

2. Results and proofs. The following is the main result of this paper.

TheOrem 1. Let $\mathcal{A}$ and $\mathcal{B}$ be unital $\mathrm{C}^{*}$-algebras with units $I$ and $I^{\prime}$, respectively. Let $\Phi: \mathcal{A} \rightarrow \mathcal{B}$ be a surjective map with $\Phi(I)-\Phi(0) \in \mathcal{Z}(\mathcal{B})$. Then $w(\Phi(A)-\Phi(B))=w(A-B)$ for all $A, B \in \mathcal{A}$ if and only if there is a central projection $P \in \mathcal{A}$ with $\Phi(P)$ a central projection in $\mathcal{B}$, a $\mathrm{C}^{*}$ isomorphism $\Phi_{1}: P \mathcal{A} P \rightarrow \Phi(P) \mathcal{B} \Phi(P)$, a conjugate $\mathrm{C}^{*}$-isomorphism $\Phi_{2}$ : $(I-P) \mathcal{A}(I-P) \rightarrow\left(I^{\prime}-\Phi(P)\right) \mathcal{B}\left(I^{\prime}-\Phi(P)\right)$, and an element $S$ and a central unitary element $U$ in $\mathcal{B}$ such that

$$
\Phi(A)=U\left(\Phi_{1}(P A P)+\Phi_{2}((I-P) A(I-P))\right)+S
$$

for all $A \in \mathcal{A}$.

To prove Theorem 1 , we need several lemmas. Denote by $B_{1}(\mathcal{A}, w)$ the set $\{A \in \mathcal{A} \mid w(A) \leq 1\}$. The first lemma characterizes the extreme points of $B_{1}(\mathcal{A}, w)$; its proof is omitted since it is similar to that of $[4$, Theorem 1 and 2].

LEMMA 2. Every extreme point of $B_{1}(\mathcal{A}, w)$ is a partial isometric element $U$ in $\mathcal{A}$ with $\left(I-U^{*} U\right) \mathcal{A}\left(I-U U^{*}\right)=\{0\}$. Moreover, $I$ is an extreme point of $B_{1}(\mathcal{A}, w)$.

LEMMA 3. Let $\Phi: \mathcal{A} \rightarrow \mathcal{B}$ be an additive map such that $w(\Phi(A)) \leq w(A)$ for every $A$ in $\mathcal{A}$ and let $\lambda$ be a given scalar. Then

(i) $\Phi(\lambda I)=\lambda I^{\prime}$ implies that for every self-adjoint element $A \in \mathcal{A}$, there is a self-adjoint element $C \in \mathcal{B}$ such that $\Phi(i \lambda A)=i \lambda C$;

(ii) $\Phi(\lambda I)=\bar{\lambda} I^{\prime}$ implies that for every self-adjoint element $A \in \mathcal{A}$, there is a self-adjoint element $C \in \mathcal{B}$ such that $\Phi(i \lambda A)=\overline{i \lambda} C$.

Proof. It is easy to see that (ii) follows from (i). Indeed, if $\Phi(\lambda I)=\bar{\lambda} I^{\prime}$, then $\Psi$ satisfies the condition in (i), where $\Psi(\cdot)=\Phi(\cdot)^{*}$. So to each selfadjoint element $A \in \mathcal{A}$ there corresponds a self-adjoint element $C \in \mathcal{B}$ such that $\Psi(i \lambda A)=i \lambda C$. Consequently, $\Phi(i \lambda A)=\overline{i \lambda} C$.

To prove (i), we suppose that $|\lambda|=1$ and $w(A)=1$. Write $\Phi(i \lambda A)=$ $\lambda(B+i C)$, where $B, C \in \mathcal{B}$ are self-adjoint. We show that $B=0$. To do this, we only need to prove that the spectral radius of $B$ is 0 since $B$ is self-adjoint. If this is not true, take a nonzero number $\beta$ from the spectrum of $B$. If $\beta>0$, then, for sufficiently large $n$,

$$
\begin{aligned}
w(\lambda(i A+n I)) & =w(i A+n I)=\left(1+n^{2}\right)^{1 / 2} \\
& <\beta+n \leq w\left(B+n I^{\prime}\right) \leq w\left(B+i C+n I^{\prime}\right) \\
& =w\left(\lambda(B+i C)+\lambda n I^{\prime}\right)=w(\Phi(i \lambda A+\lambda n I)) \leq w(\lambda(i A+n I)),
\end{aligned}
$$


which is a contradiction. If $\beta<0$, then considering $-\lambda A$ will also lead to a contradiction.

Lemma 4. If an additive surjective mapping $\Phi: \mathcal{A} \rightarrow \mathcal{B}$ preserves numerical radius and if $\Phi(\lambda I)=\lambda I^{\prime}$ for every scalar $\lambda$, then $\Phi$ is a $\mathrm{C}^{*}$ isomorphism.

Proof. We prove the lemma by checking several claims.

Claim 1. $\Phi$ preserves numerical range, that is, $W(\Phi(A))=W(A)$ for every $A \in \mathcal{A}$. Consequently, for every self-adjoint element $A \in \mathcal{A}, \Phi(A)$ is self-adjoint and there is a self-adjoint element $C \in \mathcal{B}$ such that $\Phi(i A)=i C$.

Assume that there exists a $\nu \in \mathbb{C}$ such that $\nu \in W(\Phi(A)) \backslash W(A)$. Then there is a circle with sufficiently large radius and centered at a certain $\lambda \in \mathbb{C}$ such that $W(A)$ lies inside the circle, but $\nu$ lies outside it. Hence

$$
w(A-\lambda I)<|\nu-\lambda| \leq w\left(\Phi(A)-\lambda I^{\prime}\right)=w(A-\lambda I),
$$

which is a contradiction. So $W(\Phi(A)) \subseteq W(A)$. Applying the argument to $\Phi^{-1}$, we see that $W(A) \subseteq W(\Phi(A))$. Hence, $W(\Phi(A))=W(A)$ for all $A \in \mathcal{A}$.

Claim 2. $\Phi$ is linear.

Let $A \in \mathcal{A}$ be a self-adjoint element. By Claim $1, W((1+i) A)=$ $W(\Phi(A)+\Phi(i A))$ and $\Phi(i A)=i C$ for some self-adjoint element $C \in \mathcal{B}$; consequently, $W((1+i) A)=W(\Phi(A)+i C)$. Then for every state $\tau_{\mathcal{B}}$ on $\mathcal{B}$, there is a state $\tau_{\mathcal{A}}$ on $\mathcal{A}$ such that $\tau_{\mathcal{A}}((1+i) A)=\tau_{\mathcal{B}}(\Phi(A)+i C)$. Since $A, \Phi(A), C$ are self-adjoint, $\tau_{\mathcal{B}}(\Phi(A))=\tau_{\mathcal{A}}(A)=\tau_{\mathcal{B}}(C)$. Thus $\Phi(i A)=i \Phi(A)$. Note that $\Phi$ is real-linear and every element $A$ in $\mathcal{A}$ can be written as $B+i C$ with $B, C$ being self-adjoint. So $\Phi$ is linear.

Claim 3. $\Phi$ is a $\mathrm{C}^{*}$-isomorphism.

Apply [2, Theorem 3] directly.

Lemma 5. If an additive surjective mapping $\Phi: \mathcal{A} \rightarrow \mathcal{B}$ preserves numerical radius and if $\Phi(\lambda I)=\bar{\lambda} I^{\prime}$ for every scalar $\lambda$, then $\Phi$ is a conjugate $\mathrm{C}^{*}$-isomorphism.

Proof. Define $\Psi: \mathcal{A} \rightarrow \mathcal{B}$ by $\Psi(A)=\Phi(A)^{*}$. Then $\Psi$ satisfies the conditions in Lemma 4 . So $\Psi$ is a $\mathrm{C}^{*}$-isomorphism between $\mathcal{A}$ and $\mathcal{B}$. As a consequence, $\Phi$ is a conjugate $\mathrm{C}^{*}$-isomorphism.

Now we turn to the proof of our main result.

Proof of Theorem 1. If $\Phi$ is a $\mathrm{C}^{*}$-isomorphism then the composition $\tau \circ \Phi$ is a state on $\mathcal{A}$ for every state $\tau$ on $\mathcal{B}$. Hence $\Phi$ preserves numerical radius. It is easy to see that every conjugate $\mathrm{C}^{*}$-isomorphism also preserves numerical radius. Also note that multiplying by a central unitary does not change 
the numerical radius [2]. So the maps of the form described in the theorem preserve numerical radius distance. Our main work is to check the "only if" part.

Let $\Psi(A)=\Phi(A)-\Phi(0)$. Then $\Psi(0)=0$ and $w(\Psi(A))=w(A)$ for every $A \in \mathcal{A}$. It follows from the Mazur-Ulam theorem [6] that $\Psi$ is real-linear. Consequently, we may, in what follows, assume that $\Phi$ itself is real-linear and preserves numerical radius, with $\Phi(I)$ in the center of $\mathcal{B}$. Since $\Phi(I)$ is also an extreme point of $B_{1}(\mathcal{B}, w)$, it is easily seen from Lemma 2 that $\Phi(I)$ is unitary. Thus, $(\Phi(I))^{-1}$ is a central unitary element and, by [2], $\Psi=(\Phi(I))^{-1} \Phi$ is numerical radius preserving. Therefore, we may assume further that $\Phi(I)=I^{\prime}$, and then prove that there is a central projection $P \in \mathcal{A}$ with $\Phi(P)$ a central projection in $\mathcal{B}$, a $\mathrm{C}^{*}$-isomorphism $\Phi_{1}: P \mathcal{A} P \rightarrow$ $\Phi(P) \mathcal{B} \Phi(P)$ and a conjugate $\mathrm{C}^{*}$-isomorphism $\Phi_{2}:(I-P) \mathcal{A}(I-P) \rightarrow$ $\left(I^{\prime}-\Phi(P)\right) \mathcal{B}\left(I^{\prime}-\Phi(P)\right)$ such that $\Phi(A)=\Phi_{1}(P A P)+\Phi_{2}((I-P) A(I-P))$ for every $A \in \mathcal{A}$.

Claim 1. $\Phi(i I)=i\left(2 Q-I^{\prime}\right)$ for some central projection $Q$ in $\mathcal{B}$.

By Lemma $3, \Phi(i I)=i C$ for some self-adjoint $C$. Thus $\Phi(i I) \Phi(i I)^{*}=$ $\Phi(i I)^{*} \Phi(i I)=-\Phi(i I)^{2}$. On the other hand, by Lemma $2, i I$ is an extreme point of $B_{1}(\mathcal{A}, w)$. Since $\Phi$ is real-linear and preserves numerical radius, $\Phi(i I)$ is an extreme point of $B_{1}(\mathcal{B}, w)$. By Lemma 2 again, $-\Phi(i I)^{2}$ is a projection with $\left(I^{\prime}+\Phi(i I)^{2}\right) \mathcal{B}\left(I^{\prime}+\Phi(i I)^{2}\right)=0$. Hence $\Phi(i I)^{2}=-I^{\prime}$. Let $Q=\left(I^{\prime}-i \Phi(i I)\right) / 2$; then $Q$ is a projection in $\mathcal{B}$ with $\Phi(i I)=i\left(2 Q-I^{\prime}\right)$.

In the following, we show that $\Phi(i I)$ is contained in the center of $\mathcal{B}$, and consequently, $Q$ is a central projection. For any nonzero self-adjoint element $B$ in $\mathcal{B}$, Lemma 3 applied to $\Phi^{-1}$ yields a self-adjoint $A \in \mathcal{A}$ such that $\Phi(i A)=i B$. It is easy to see that either $w(i I+i A)=1+w(A)$ or $w(i I-i A)=1+w(A)$. Then either $w(\Phi(i I)+i B)=1+w(B)$ or $w(\Phi(i I)-i B)$ $=1+w(B)$. If $w(\Phi(i I)+i B)=1+w(B)$, then there is a state $\tau$ on $\mathcal{B}$ such that $|\tau(\Phi(i I))+\tau(i B)|=1+w(B)$. It follows that $|\tau(\Phi(i I))|=1$ and $|\tau(i B)|=w(B) \neq 0$. Thus the state $\tau$ satisfies $\tau\left((\Phi(i I))^{2}\right)=(\tau(\Phi(i I)))^{2}$ $=-1$ and $|\tau(i B)|=w(B) \neq 0$. So by [5, p. 292, 4.6.32], $\Phi(i I)$ lies in the center of $\mathcal{B}$. The case that $w(\Phi(i I)-i B)=1-w(B)$ can be dealt with similarly.

Claim 2. $\Phi$ preserves self-adjoint operators in both directions.

Since $\Phi$ is invertible and $\Phi^{-1}$ has the same properties as $\Phi$, we only check that $\Phi$ sends self-adjoint elements to self-adjoint elements. Let $A$ be an arbitrary self-adjoint element in $\mathcal{A}$ with $w(A)=1$. By Claim $1, \Phi(A)=$ $Q \Phi(A) Q+\left(I^{\prime}-Q\right) \Phi(A)\left(I^{\prime}-Q\right)$. Note that $Q \Phi(\cdot) Q: \mathcal{A} \rightarrow Q \mathcal{B} Q$ satisfies $w(Q \Phi(T) Q) \leq w(T)$ for every $T \in \mathcal{A}$ and $Q \Phi(i I) Q=i Q$. Using Lemma 3, we see that $Q \Phi(A) Q$ is self-adjoint. Similarly, since $\left(I^{\prime}-Q\right) \Phi(\cdot)\left(I^{\prime}-Q\right)$ : 
$\mathcal{A} \rightarrow\left(I^{\prime}-Q\right) \mathcal{B}\left(I^{\prime}-Q\right)$ satisfies $w\left(\left(I^{\prime}-Q\right) \Phi(T)\left(I^{\prime}-Q\right)\right) \leq w(T)$ for every $T \in \mathcal{A}$ and $\left(I^{\prime}-Q\right) \Phi(i I)\left(I^{\prime}-Q\right)=-i\left(I^{\prime}-Q\right)$, by Lemma 3 again, $\left(I^{\prime}-Q\right) \Phi(A)\left(I^{\prime}-Q\right)$ is self-adjoint. Therefore, $\Phi(A)$ is self-adjoint.

Claim 3. $\Phi$ preserves projections in both directions.

Just as in the proof of Claim 2, we only need to show that $\Phi(P)$ is a projection whenever $P \in \mathcal{A}$ is. First, we show that $\Phi$ preserves positive elements. Let $A \in \mathcal{A}$ be positive with $w(A) \leq 1$. Then $\|A-I\| \leq 1$, and therefore $w\left(\Phi(A)-I^{\prime}\right)=w(A-I) \leq 1$. It turns out that $\Phi(A)$ can have no strictly negative spectrum since $\Phi(A)$ is self-adjoint. So $\Phi(A)$ is positive.

By [4, Theorem 4], the set of all extreme points of the positive portion of $B_{1}(\mathcal{A}, w)$ is the set of all projections in $\mathcal{A}$. Now, since the real-linear map $\Phi$ preserves numerical radius and positive elements, $\Phi$ preserves the extreme point of the positive portion of $B_{1}(\mathcal{A}, w)$. Therefore $\Phi$ preserves projections.

Claim 4. There is a central projection $P \in \mathcal{A}$ such that $\Phi(P)=Q$.

By Claim 3, there is a projection $P \in \mathcal{A}$ such that $\Phi(P)=Q$. We will show that $P$ lies in the center of $\mathcal{A}$. It is easy to see that $\Phi(2 P-I)=-i \Phi(i I)$. Note that for every self-adjoint $A \in \mathcal{A}$, either $w(\Phi(i I)+i \Phi(A))=1+w(\Phi(A))$ or $w(\Phi(i I)-i \Phi(A))=1+w(\Phi(A))$. Hence either

$$
\begin{aligned}
w(2 P-I+A) & =w(\Phi(2 P-I)+\Phi(A))=w(-i \Phi(i I)+\Phi(A)) \\
& =w(\Phi(i I)+i \Phi(A))=1+w(\Phi(A))=1+w(A)
\end{aligned}
$$

or

$$
\begin{aligned}
w(2 P-I-A) & =w(\Phi(2 P-I)-\Phi(A))=w(-i \Phi(i I)-\Phi(A)) \\
& =w(\Phi(i I)-i \Phi(A))=1+w(\Phi(A))=1+w(A) .
\end{aligned}
$$

It is also easily seen that, in either case, there is a state $\tau$ on $\mathcal{A}$ such that $|\tau(2 P-I)|=1$ and $|\tau(A)|=w(A) \neq 0$. Thus $\tau\left((2 P-I)^{2}\right)=(\tau(2 P-I))^{2}$ and $|\tau(A)|=w(A) \neq 0$. So, by [5, p. 292, 4.6.32], $2 P-I$ lies in the center of $\mathcal{B}$.

Claim 5. $\Phi_{1}=\left.\Phi\right|_{P \mathcal{A} P}$ is a $\mathrm{C}^{*}$-isomorphism between PAP and $Q \mathcal{B} Q$, and $\Phi_{2}=\left.\Phi\right|_{(I-P) \mathcal{A}(I-P)}$ is a conjugate $\mathrm{C}^{*}$-isomorphism of $(I-P) \mathcal{A}(I-P)$ and $\left(I^{\prime}-Q\right) \mathcal{B}\left(I^{\prime}-Q\right)$.

It is easily checked that $\Phi_{1}(\lambda P)=\lambda Q$ and $\Phi_{2}(\lambda(I-P))=\bar{\lambda}\left(I^{\prime}-Q\right)$ for every complex number $\lambda$. Now the proof is completed by using Lemmas 4 and 5 .

For the von Neumann algebra case, we have the following corollaries.

Corollary 6. Let $\mathcal{A}$ and $\mathcal{B}$ be von Neumann algebras, and let $\Phi: \mathcal{A} \rightarrow$ $\mathcal{B}$ be a surjective map with $\Phi(I)-\Phi(0)=I^{\prime}$. Then $w(\Phi(A)-\Phi(B))=$ $w(A-B)$ for all $A, B \in \mathcal{A}$ if and only if there is an element $S$ in $\mathcal{B}$, and 
there are four central projections $P_{1}, P_{2}, P_{3}, P_{4}$ in $\mathcal{A}$ with $P_{1}+P_{2}+P_{3}+$ $P_{4}=I$ and $\Phi\left(P_{i}\right) \in \mathcal{Z}(\mathcal{B})(i=1, \ldots, 4), a{ }^{*}$-isomorphism $\Phi_{1}: P_{1} \mathcal{A} P_{1} \rightarrow$ $\Phi\left(P_{1}\right) \mathcal{B} \Phi\left(P_{1}\right)$, a conjugate ${ }^{*}$-isomorphism $\Phi_{2}: P_{2} \mathcal{A} P_{2} \rightarrow \Phi\left(P_{2}\right) \mathcal{B} \Phi\left(P_{2}\right)$, a ${ }^{*}$-anti-isomorphism $\Phi_{3}: P_{3} \mathcal{A} P_{3} \rightarrow \Phi\left(P_{3}\right) \mathcal{B} \Phi\left(P_{3}\right)$, and a conjugate ${ }^{*}$-antiisomorphism $\Phi_{4}: P_{4} \mathcal{A} P_{4} \rightarrow \Phi\left(P_{4}\right) \mathcal{B} \Phi\left(P_{4}\right)$ such that

$$
\Phi(A)=\Phi_{1}\left(P_{1} A P_{1}\right)+\Phi_{2}\left(P_{2} A P_{2}\right)+\Phi_{3}\left(P_{3} A P_{3}\right)+\Phi_{4}\left(P_{4} A P_{4}\right)+S
$$

for all $A \in \mathcal{A}$.

Proof. We only need to check the "only if" part. By Theorem 1, there is a central projection $P \in \mathcal{A}$ with $\Phi(P) \in \mathcal{Z}(\mathcal{B})$, a $\mathrm{C}^{*}$-isomorphism $\Phi_{1}$ : $P \mathcal{A} P \rightarrow \Phi(P) \mathcal{B} \Phi(P)$, a conjugate $\mathrm{C}^{*}$-isomorphism $\Phi_{2}:(I-P) \mathcal{A}(I-P) \rightarrow$ $\left(I^{\prime}-\Phi(P)\right) \mathcal{B}\left(I^{\prime}-\Phi(P)\right)$ and an element $S \in \mathcal{B}$ such that $\Phi(A)=\Phi_{1}(P A P)+$ $\Phi_{2}((I-P) A(I-P))+S$ for every $A \in \mathcal{A}$. Note that every $\mathrm{C}^{*}$-isomorphism between two von Neumann algebras is the direct sum of a ${ }^{*}$-isomorphism and a *-anti-isomorphism (see [7]). That is, there is a central projection $R$ in $\Phi(P) \mathcal{B} \Phi(P)$ such that $\Phi(\cdot) R$ is a *-isomorphism and $\Phi(\cdot)(\Phi(P)-R)$ is a *anti-isomorphism. It is known from [4] that every $\mathrm{C}^{*}$-isomorphism preserves commutativity. Hence there are orthogonal projections $P_{1}, P_{2}$ in $\mathcal{Z}(A)$ such that $P_{1}+P_{2}=P, \Phi\left(P_{1}\right)=R$ and $\Phi\left(P_{2}\right)=\Phi(P)-R$. So $\left.\Phi\right|_{P_{1} \mathcal{A} P_{1}}: P_{1} \mathcal{A} P_{1} \rightarrow$ $\Phi\left(P_{1}\right) \mathcal{B} \Phi\left(P_{1}\right)$ is a ${ }^{*}$-isomorphism and $\left.\Phi\right|_{P_{2} \mathcal{A} P_{2}}: P_{2} \mathcal{A} P_{2} \rightarrow \Phi\left(P_{2}\right) \mathcal{B} \Phi\left(P_{2}\right)$ is a ${ }^{*}$-anti-isomorphism. Similarly, there are orthogonal projections $P_{3}, P_{4} \in$ $\mathcal{Z}(\mathcal{A})$ such that $P_{3}+P_{4}=I-P,\left.\Phi\right|_{P_{3} \mathcal{A} P_{3}}: P_{3} \mathcal{A} P_{3} \rightarrow \Phi\left(P_{3}\right) \mathcal{B} \Phi\left(P_{3}\right)$ is a conjugate ${ }^{*}$-isomorphism and $\left.\Phi\right|_{P_{4} \mathcal{A} P_{4}}: P_{4} \mathcal{A} P_{4} \rightarrow \Phi\left(P_{4}\right) \mathcal{B} \Phi\left(P_{4}\right)$ is a conjugate *-anti-isomorphism. The proof is finished.

Corollary 7. Let $\mathcal{A}$ and $\mathcal{B}$ be von Neumann algebras with $\mathcal{B}$ a factor, and let $\Phi: \mathcal{A} \rightarrow \mathcal{B}$ be a surjective map with $\Phi(I)-\Phi(0)=I^{\prime}$. Then $w(\Phi(A)-$ $\Phi(B))=w(A-B)$ for all $A, B \in \mathcal{A}$ if and only if there is an element $S$ in $\mathcal{B}$ such that $\Phi(\cdot)-S$ is either $a^{*}$-isomorphism, $a{ }^{*}$-anti-isomorphism, a conjugate ${ }^{*}$-isomorphism, or a conjugate ${ }^{*}$-anti-isomorphism.

In the following, we characterize 2-local numerical radius isometry on $\mathrm{C}^{*}$-algebras. A map $\Phi: \mathcal{A} \mapsto \mathcal{B}$ is called a numerical radius isometry if $\Phi$ is linear, surjective and numerical radius preserving (i.e. $w(\Phi(A))=w(A)$ for every $A \in \mathcal{A}) ; \Phi$ is called a 2-local numerical radius isometry if for any two elements $A, B \in \mathcal{A}$, there is a numerical radius isometry $\Phi_{A, B}$ such that $\Phi(A)=\Phi_{A, B}(A)$ and $\Phi(B)=\Phi_{A, B}(B)$.

TheOREM 8. Let $\mathcal{A}, \mathcal{B}$ be unital $\mathrm{C}^{*}$-algebras with units $I, I^{\prime}$ respectively. Let $\Phi: \mathcal{A} \rightarrow \mathcal{B}$ be a surjective 2-local numerical radius isometry. Then $\Phi$ is a multiple of a $\mathrm{C}^{*}$-isomorphism by a fixed unitary element in the center of $\mathcal{B}$.

Proof. By the 2-local property of $\Phi$, it follows that $w(\Phi(A)-\Phi(B))=$ $w(A-B)$ for every $A, B \in \mathcal{A}$ and $\Phi(0)=0$. Using the Mazur-Ulam theorem, 
we deduce that $\Phi$ is real-linear. Let $A$ be an element in $\mathcal{A}$. For any scalar $\lambda \in$ $\mathbb{C}$, there is a numerical radius isometry $\Phi_{A, \lambda A}$ such that $\Phi_{A, \lambda A}(A)=\Phi(A)$ and $\Phi_{A, \lambda A}(\lambda A)=\Phi(\lambda A)$. Since $\Phi_{A, \lambda A}$ is linear, we have $\Phi(\lambda A)=\lambda \Phi(A)$. Hence $\Phi$ is in fact linear. Now the proof is finished by using [2, Theorem 3].

\section{References}

[1] Z. F. Bai and J. C. Hou, Numerical radius distance preserving maps on $\mathcal{B}(H)$, Proc. Amer. Math. Soc., to appear.

[2] J. T. Chan, Numerical radius preserving operators on $C^{*}$-algebras, Arch. Math. (Basel) 70 (1998), 486-488.

[3] J. L. Cui and J. C. Hou, Non-linear numerical radius isometries on atomic nest algebras and diagonal algebras, J. Funct. Anal. 206 (2004) 414-448.

[4] R. V. Kadison, Isometries of operator algebras, Ann. of Math. 54 (1951), 325-338.

[5] R. V. Kadison and J. R. Ringrose, Fundamentals of the Theory of Operator Algebras, Volume I: Elementary Theory, Grad. Stud. in Math. 15, Amer. Math. Soc., 1997.

[6] S. Mazur et S. Ulam, Sur les transformations isométriques d'espaces vectoriels normés, C. R. Acad. Sci. Paris 194 (1932), 946-948.

[7] E. Størmer, On the Jordan structure of $C^{*}$-algebras, Trans. Amer. Math. Soc. 120 (1965), 438-447.

[8] Z. X. Wan, Geometry of Matrices, World Sci., 1996.

School of Science

Xi'an Jiaotong University

Xi'an, 710049, P.R. China

Department of Mathematics

Shanxi Teachers University

Linfen, 041004, P.R. China.

E-mail: jhou@dns.sxtu.edu.cn
Department of Mathematics

Shanxi University

Taiyuan, 030000, P.R. China

Received March 10, 2003

Revised version August 28, 2003 